\title{
REVIEW
}

\section{Is Community-Acquired Methicillin-Resistant Staphylococcus aureus Coverage Needed for Cellulitis?}

Michael Horseman · John D. Bowman

To view enhanced content go to www.infectiousdiseases-open.com Received: September 19, 2013 / Published online: November 12, 2013

(c) The Author(s) 2013. This article is published with open access at Springerlink.com

\section{ABSTRACT}

Methicillin-resistant Staphylococcus aureus (MRSA) has become the dominant strain of Staphylococcus aureus in many communities of the United States. As a result, many clinicians are now empirically covering for this pathogen in the treatment of various skin and soft-tissue infections. Should this practice apply to cellulitis? In order to answer this question, we defined cellulitis and reviewed the pathogenesis, microbiology, and current studies of inpatient and outpatient antimicrobial therapy. The current evidence suggests empirical MRSA coverage for community-acquired cellulitis may not be necessary in non-purulent (non-suppurative) forms of this infection. Most cases are non-purulent and not amenable to

M. Horseman · J. D. Bowman ( $₫)$

Department of Pharmacy Practice, Rangel College of Pharmacy, Texas A\&M Health Science Center, MSC 131, 1010 West Avenue B, Kingsville, TX 78363-8202, USA

e-mail: bowman@pharmacy.tamhsc.edu

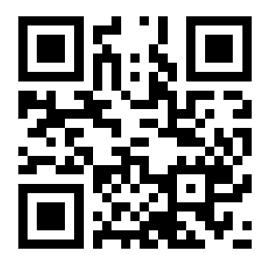

Enhanced content for this article is

available on the journal web site:

www.infectiousdiseases-open.com culture although antibody studies indicate streptococci are the most common etiologic agents. Current studies of antimicrobial therapy tend to agree with this finding. Empirical betalactam therapy directed primarily at streptococci appears sufficient for non-purulent cellulitis regardless of the prevalence of MRSA in the community.

Keywords: Antibiotic; Cellulitis; Infectious diseases; Methicillin-resistant Staphylococcus aureus; Skin and soft-tissue infection; Streptococci

\section{INTRODUCTION}

In what situations or under what circumstances should coverage for community-acquired methicillin-resistant Staphylococcus aureus (CAMRSA) be included in empirical regimens for cellulitis? Published guidelines offer different recommendations. In addition, there are differences in definitions for cellulitis. We will review what has been published since the 2005 Infectious Diseases Society of America (IDSA) guideline. 
The 2013 Sanford guide recommends only empirical streptococcal coverage for cellulitis of the extremities in non-diabetics [1]. MRSA coverage is recommended only for severe disease in diabetics and facial cellulitis. The Johns Hopkins ABX Guide generally concurs with the Sanford guide in emphasizing antistreptococcal coverage but recommends MRSA coverage for hospitalized patients (intravenous clindamycin, vancomycin, linezolid, daptomycin, ceftaroline, or telavancin) regardless of the presence of diabetes [2].

The IDSA guideline for erysipelas or cellulitis recommends "dicloxacillin, cephalexin, clindamycin, or erythromycin, unless streptococci or staphylococci resistant to these agents are common in the community" [3]. The IDSA guidelines were published in 2005 and an update will not be ready until late 2013 [4].

The more recent (published 2011) IDSA guidelines for MRSA recommend empirical (MRSA) coverage only for purulent cellulitis [5]. In 2007, the Centers for Disease Control published similar guidelines for skin and softtissue infections that included endorsement by IDSA and the American Medical Association [6]. Empirical MRSA coverage for non-purulent cellulitis is not recommended unless a therapeutic failure has occurred. These guidelines also suggest that empirical (MRSA) coverage for complicated skin and soft-tissue infections be considered in hospitalized patients.

MRSA has become common in the United States and is more prevalent than methicillinsensitive Staphylococcus aureus (MSSA) in many communities [7]. Many, if not most physicians, routinely cover for MRSA using trimethoprim/ sulfamethoxazole (TMP/SMX), clindamycin, doxycycline or fluoroquinolones in patients with cellulitis [8]. Some authors advocate empirical coverage of cellulitis when the skin is intact [9]. Others suggest that empirical therapy for CAMRSA be limited to seriously ill patients or those who have failed initial empirical therapy [10]. Still others recommend such coverage when the community prevalence is high, such as greater than $10-15 \%[7,11]$. Is that appropriate in 2013? Should diabetics with cellulitis always receive empirical coverage for MRSA?

\section{METHODS}

PubMed was searched for the terms "cellulitis," "MRSA," "skin and soft tissue infection," "community acquired staphylococcus" and combinations of these terms during the month of May, 2013. The results were narrowed by omitting articles not in English and those with terms including ophthalmic, systemic, case studies, hospitalized, and purulent. Additional articles were added in October as a result of reviewer's comments.

The analysis in this article is based on previously conducted studies, and does not involve any new studies of human or animal subjects performed by any of the authors.

\section{WHAT IS CELLULITIS?}

What is and what is not cellulitis is important in determining a possible microbiological etiology and treatment. Unfortunately, cellulitis is often used to describe a broad group of superficially similar (e.g., diffuse and spreading) but often histologically distinct skin infections. The International Classification of Diseases version 9 (ICD-9) creates further confusion by combining cellulitis and abscess under a single code [12].

Cellulitis, as defined in the 2005 IDSA skin and soft-tissue infection guideline, is a diffuse 
spreading infection with inflammation of the deeper dermis and subcutaneous fat. It excludes "infections associated with underlying suppurative foci, such as cutaneous abscesses, necrotizing fasciitis, septic arthritis, and osteomyelitis" [3]. This definition is largely histologic and excludes underlying complicating or complex lesions. It delineates cellulitis as the primary focus of infection and not one resulting from contiguous extension. This definition does not, however, exclude the possibility of suppurative complications from cellulitis.

Cellulitis is characterized by rapidly spreading areas of edema, redness, and heat, sometimes accompanied by lymphangitis and inflammation of the regional lymph nodes. Other manifestations such as vesicles, bullae, and petechiae or ecchymoses may develop on the inflamed skin. The affected integument may eventually develop a pitting orange peel appearance. Systemic manifestations are usually mild, but fever, tachycardia, confusion, hypotension, and leukocytosis may be present and occur hours before the skin abnormalities appear. Vesicles and bullae filled with clear fluid are common. The presence of severe pain, violaceous blisters or bullae, and petechiae or ecchymoses, if widespread or associated with systemic toxicity, may signal a deeper infection such as necrotizing fasciitis $[3,12,13]$.

The etiologic agent of cellulitis is believed to be streptococci or Staphylococcus aureus in most cases but can vary depending on extenuating factors. These extenuating factors include physical activities, trauma, water contact, injection drug use or abuse and animal, insect, or human bites. Cellulitis that is diffuse or unassociated with a defined portal is believed to be caused by Streptococcus species [3, 12-16].

The general term cellulitis has also been applied to several diffuse spreading skin infections. Some of these do not meet the IDSA Guidelines definition. When used as a general term, the word cellulitis is usually preceded by some type of adjective such as purulent, suppurative, non-purulent, nonsuppurative, necrotizing, synergistic necrotizing, periorbital, buccal, and perianal. Other forms of "cellulitis" are followed by "with" and a noun. These include cellulitis with abscess, cellulitis with drainage, and cellulitis with ulcer $[12,16,17]$.

Several of these descriptors really point to a much more complex infection than cellulitis. Suppurative or purulent cellulitis indicates the presence of pus in the form of an exudate and in the absence of a drainable abscess. Nonsuppurative or non-purulent cellulitis indicates the absence of both an exudate and abscess. Erysipelas is another skin and soft-tissue infection commonly classified as cellulitis but is more superficial affecting the upper dermis. Although both infections are generally similar in surface appearance, the border of erysipelas is sharply demarcated and raised whereas the border of cellulitis is diffuse and flush with surrounding skin. Systemic effects as described above may also occur with erysipelas. According to some authors, erysipelas and cellulitis may coexist at the same site making differentiation difficult. Erysipelas also usually affects children and the elderly whereas cellulitis occurs in all age groups. The etiologic agent of erysipelas is believed to be almost always streptococci $[3,12,15,17]$.

Two outdated descriptors often applied to skin and soft-tissue infections in general are uncomplicated and complicated. No form of cellulitis using the IDSA guideline definition would be complicated. ICD-9 coding does not always discriminate between these two outdated descriptors. Complicated skin and soft-tissue infections are considered infected 
burns, deep-tissue infections, major abscesses, infected ulcers, and perirectal abscesses [18].

Some skin conditions mimic cellulitis and have been referred to as "pseudo-cellulitis" [19]. These include allergic dermatitis, contact dermatitis, thrombophlebitis and DVT, panniculitis and erythema migrans.

\section{PATHOGENESIS AND MICROBIOLOGY}

There is relatively little information in the literature about the pathogenesis of cellulitis. Most cases result from microbial invasion through a breach in the skin. Lacerations, bite or puncture wounds, scratches, instrumentation (e.g., needles), pre-existing skin conditions or infections (e.g., chicken pox, impetigo, or ulcer), burns, and surgery are more among the common portals of entry. In many cases the skin breaks are not clinically apparent [3, 13, 15]. Bacteremia may contribute to some cases of cellulitis. The most common site of infection is the lower extremities (up to $70-88 \%$ of cases) $[3,13,14,20]$. Fissured webbing of the toes from maceration, dermatophyte infection, or inflammatory dermatoses is believed to contribute in many cases $[3,13,15,21]$.

A number of risk factors have been identified for both initial and recurrent episodes of lower extremity cellulitis. These include obesity, chronic edema from venous insufficiency or lymphatic obstruction, previous cellulitis, saphenectomy, and skin barrier disruption especially web toe intertrigo [3, 13, 15, 21-24]. Other putative factors include smoking, previous surgery, and previous antibiotic use [22].

Edema is a major contributor to the development of cellulitis by creating small, unapparent breaks in the skin. Swollen cutaneous surfaces are also taut, fragile, and more easily disrupted with minor trauma than normal skin. The role of lymphatic obstruction may relate to the inability to clear the pathogen. Venous insufficiency may also cause "venous eczema" or stasis dermatitis which could disrupt the cutaneous barrier. More obvious breaches in the form of stasis ulcers are also possible. The role of obesity may be difficult to separate from edema since the two often go hand in hand. Adipose tissue, however, can compress lymphatic channels and impair lymphatic flow. Obesity may also increase skin fragility and decrease hygiene levels [13].

Groups A, B, C, and G streptococci and Staphylococcus aureus are considered to be the most common etiologic agents of cellulitis [3, 13, 15, 16].

Depending on extenuating factors, other microbes can cause cellulitis. These include Vibrio and Aeromonas species associated with exposure to marine and freshwater environments, respectively, Pasteurella multocida associated with carnivore (especially cat) bites, Pseudomonas aeruginosa associated with neutropenia, and Erysipelothrix rhusiopathiae associated with the handling of seafood or meat. Cryptococcus neoformans may cause cellulitis in patients with defective cellmediated immunity $[3,13,15,16,25]$.

Biopsy of skin with cellulitis has shown dilated lymphatics and capillaries, marked dermal edema, and primarily neutrophilic infiltration, either diffusely within the dermis or concentrated around vessels [13]. The bacterial burden from central and peripheral biopsy is usually low suggesting an exaggerated inflammatory response to low concentrations of microorganisms or possibly their export products [26].

It has been suggested that exotoxins elaborated by streptococci or staphylococci are 
really the primary mediators of inflammation. This theory proposes that immune responses to exotoxins are responsible for most of the tissue effects seen in cellulitis as opposed to direct cytotoxic effects of the exotoxins. In other words, the exotoxin would function as a superantigen $[13,27]$.

\section{CULTURE ETIOLOGY}

Most cases of cellulitis are not amenable to identification of a pathogen [3, 7, 13, 15]. Microbiological cultures are usually negative for the majority of cases in which cultures are performed [8].

A study of quantitative cultures of biopsy specimens from cutaneous cellulitis found that only $28.5 \%$ and $18 \%$ of needle aspiration and punch biopsy cultures were positive, respectively [26].

Other studies have shown blood cultures were even less likely to be positive with yields $<5 \%$ [28-30]. Slightly higher yields (up to 7-10\%) have been reported for patients who had not previously received antimicrobial therapy [13]. As a result, cultures of nonsuppurative cellulitis are rarely formed, and treatment is informed by expert guidelines and clinical judgment.

Positive blood cultures are most commonly associated with streptococci [12, 13, 15]. Needle aspirations and punch biopsies are most commonly associated with Staphylococcus aureus. Other techniques for pathogen identification such as serologic and antigen studies either alone or in combination have shown a high (about 70-88\%) streptococcal predominance. These include antistreptolysin $\mathrm{O}$ (ASO), antideoxyribonuclease B (ADB), and antihyaluronidase (AHT) studies and immunofluorescent staining for streptococcal antigens of groups A, C, D, and G in skin biopsy specimens $[13,15]$.

The overall body of evidence suggests that streptococci are the most common single pathogen in cellulitis $[3,12,13,15]$. These bacteria may either cause or contribute to up to $75-90 \%$ of cases [13]. However, there are some recent reports that continue to disagree with this conclusion [9, 31]. Nevertheless, there seems to be a general agreement that cases of suppurative (or purulent) cellulitis and those associated with penetrating trauma or injection drug use are more likely to have a staphylococcal etiology $[12$, 15]. Yet, surgical drainage for purulent abscesses has long been the mainstay of therapy for such infections, most of which resolve without ancillary antimicrobial therapy [32]. The role of empirical therapy in these patients remains undetermined. Communityassociated MRSA (CAMRSA) is probably a minor contributor to non-suppurative cases of cellulitis if at all $[12,13]$.

Gunderson and Martinello conducted a systematic review of bacteremias in cellulitis and erysipelas, excluding reports of complicated cases, such as abscess, chronic diabetic infections and necrotizing infections [33]. Streptococcal species were the predominant culture finding, with $S$. aureus accounting for $15 \%$ of positive culture results. Surprisingly, Gram-negative bacteria accounted for as many cases as $S$. aureus. S. aureus was noted at similar rates in both erysipelas and cellulitis, at odds with the idea that almost all erysipelas is streptococcal.

A recent study reported that non-suppurative cellulitis may not be significantly associated with MRSA, even in areas where CAMRSA is endemic. The authors based their conclusions on the comparable low prevalence of nasal and inguinal colonization with CAMRSA in patients with cellulitis in comparison to population 
controls. The study was conducted in a region where methicillin-resistant strains were the dominant form of Staphylococcus aureus [18]. This finding is particularly important since most cases of cellulitis not amenable to routine culture are considered non-suppurative $[8,12]$. It also reinforces the recommendation against empirical coverage for MRSA in nonsuppurative cellulitis [5].

\section{STUDIES OF EMPIRICAL COVERAGE FOR CELLULITIS}

At least four trials have been published since the release of the 2005 IDSA guidelines comparing beta lactams to antimicrobial agents with activity against CAMRSA in cases of outpatient cellulitis [8, 31, 34]. Two studies [8, 34] evaluated "uncomplicated cellulitis" defined as non-purulent cellulitis or minimal purulence, not associated with ulcers or other complex lesions. Both trials excluded patients with diabetes mellitus as well as those who were immunocompromised. The third study included diabetics (36\%) as well as patients with cellulitis with ulcer and cellulitis with abscess [31].

The first trial by Madaras-Kelly et al. [34] was published in 2008. This multicenter retrospective cohort study evaluated 861 patients. Beta lactams were prescribed for 631 patients and included primarily cephalexin, dicloxacillin, and amoxicillin-clavulanate. Non-beta lactams with activity against CAMRSA were prescribed for 230 patients and included primarily clindamycin, trimethoprimsulfamethoxazole, and a fluoroquinolone (gatifloxacin or ciprofloxacin). Failure rates were 14.7 and $17.0 \%$ for the beta lactam and non-beta lactam groups, respectively (OR 0.85 ; 95\% CI 0.55-1.31). Failure rates in the non-beta lactam group were highest for trimethoprimsulfamethoxazole (18.6\%) and the fluoroquinolones (24.2\%). However, these were not statistically significantly different in comparison to other antimicrobial agents or the beta lactam class. MRSA colonization was reported $>30$ days prior to treatment in $4.3 \%$ of the non-beta lactam patients and in only $1.4 \%$ of the beta lactam patients $(p=0.014)$. This study included a few animal bites and 40\% had a defined portal of entry.

The second trial by Pallin was published in 2013 [8]. This randomized, double-blind, multicenter study evaluated 146 patients (both adults and children). Cephalexin (from $300 \mathrm{mg}$ QID to $1 \mathrm{~g}$ QID) plus placebo (control group) was administered to half of the patients (73). Cephalexin (same dose) plus trimethoprimsulfamethoxazole (from 40/200 mg QID to $160 / 800 \mathrm{mg}$ QID) was given to the other half. Clinical cure was achieved in 60 of 73 (82\%) patients in the control group and in 62 of 73 $(85 \%)$ of the interventional group $(95 \% \mathrm{CI}$ $-9.3 \%$ to $15 \% ; p=0.66)$. Colonization data was obtained from 142 patients. Three of 69 patients in the control group and 4 of 72 in the intervention group were colonized with MRSA. Colonization had no impact on outcomes $(p=0.67)$ [8].

The third trial by Khawcharoenporn and Tice [31] was published in 2010. This retrospective cohort study evaluated 405 patients at a teaching clinic of a tertiary hospital. Cephalexin was prescribed for 180 patients. Trimethoprim-sulfamethoxazole and clindamycin were prescribed for 152 patients and 40 patients, respectively. The remaining 33 patients received miscellaneous antimicrobial agents including amoxicillin-clavulanate, amoxicillin, dicloxacillin, tetracycline, doxycycline, ciprofloxacin, moxifloxacin, and 
azithromycin. Forty-four percent of patients had cellulitis with abscess, 36\% had "simple cellulitis" while the remainder had cellulitis with ulcer. Two-thirds of the patients with abscesses received incision and drainage. The success rate for trimethoprim-sulfamethoxazole was significantly higher than that for cephalexin (91\% vs. 74\%; OR 3.38; 95\% CI 1.79-6.39; $p<0.001)$. The rate for clindamycin was also higher than that for cephalexin but did not reach statistical significance (85\% vs. $74 \%$; $\mathrm{OR}=1.96 ; \quad 95 \% \quad$ CI $\quad 0.79-4.80 ; \quad p=0.22$ ) According to the authors, "The higher success rates of trimethoprim-sulfamethoxazole compared with cephalexin were consistent regardless of the presence of wound or abscess, the severity of cellulitis, or whether drainage was performed". MRSA grew from 72 of the 117 cultures of ulcers or abscesses collected from 129 patients. All 72 isolates were susceptible to trimethoprim-sulfamethoxazole. Streptococci grew from only 9 cultures [31].

A prospective trial by Jeng et al. [10] was published in 2010 and evaluated 179 inpatients with diffuse, non-culturable cellulitis. It included infections on various regions of the body with the exception of those involving periorbital, perineal, and groin regions. Most cases of cellulitis occurred on the lower extremities. All patients were assessed for streptococcal ASO and ADB antibodies. This trial was designed to evaluate the efficacy of beta lactams (primarily cefazolin 1 gm q $8 \mathrm{~h}$ ) without a comparator. One hundred and sixteen of 121 (95.8\%) evaluable patients responded to therapy including 21/23 (91\%) without evidence of streptococcal infection. Nearly $28 \%$ of the study patients had diabetes mellitus. MRSA colonization was not evaluated.

Jenkins and associates retrospectively reviewed discharged patients from a Denver hospital for 2007 using ICD-9 coding data for
SSTIs [35]. The primary outcome of interest was treatment failure. They noted that $85 \%$ of patients with cellulitis received anti-MRSA therapy, and nearly half were discharged on a regimen of TMP/SMX. The failure rate for cellulitis was $12 \%$. Most patients were treated with broad-spectrum antibacterial agents, and for a median duration of nearly 2 weeks. The authors suggested SSKI patients would be appropriate for antimicrobial stewardship programs.

Jenkins and associates [36] subsequently developed a clinical practice guideline (available as an eFigure in their article) to standardize management of cellulitis and cutaneous abscess at their hospital. Parenteral vancomycin was suggested for empirical therapy, along with alternatives to blood cultures. Patients with a discharge diagnosis of cellulitis or cutaneous abscess were compared for 1 year prior to and following implementation of the guideline. Blood culture use declined, as did the use of imaging studies for cellulitis. Vancomycin use increased while beta lactam/beta lactamase inhibitor combinations decreased. On discharge, doxycycline use increased while amoxicillin/ clavulanate use decreased. Median duration of antibiotic use decreased from 13 to 10 days. Clinical failure rates did not change.

\section{STUDY OF PROPHYLACTIC ANTIBIOTICS FOR RECURRENT CELLULITIS}

A double-blind randomized, controlled trial by Thomas et al. [37] was published in 2013. This multicenter study evaluated 274 patients with a history of at least 2 previous cases of cellulitis of the leg within the previous 3 years. One hundred and thirty-six patients received penicillin V $250 \mathrm{mg}$ bid for 12 months while 
the remaining patients received placebo. Participants were followed for 3 years. The median times to recurrence were 626 and 532 days in the penicillin and placebo groups, respectively. During the initial 12 months, 30 of the 136 prophylaxis patients had recurrence of cellulitis in comparison to 51 of the 138 placebo patients (hazard ratio 0.55 ; 95\% CI 0.35-0.86; $p=0.01$ ). Participants were excluded from the trial if they had a prior history of leg ulcer or trauma. Most had a history of edema and the mean body mass index (BMI) was slightly $>35$. Although diabetes mellitus was not an exclusion criterion for the trial, the authors did not report how many participants, if any, had this disorder. Patients with a BMI $>33$, three or more previous episodes of cellulitis, or edema had a poorer response to therapy. The authors speculated the penicillin dose may have been too low for the participants with high BMIs [37].

\section{SHOULD EMPIRICAL ANTIMICROBIAL COVERAGE FOR CELLULITIS INCLUDE AGENTS WITH ACTIVITY AGAINST MRSA?}

The question will likely be addressed with the new IDSA guideline for skin and soft-tissue infections in the fall of 2013. It is unlikely the current recommendations will change substantially if at all. Recent data has done more to reinforce these as well as those in the 2011 MRSA guideline. Therefore, for "non-suppurative cellulitis", it appears that empirical coverage for MRSA may not be warranted even in patients who are or were previously colonized (with MRSA) at the time of diagnosis, or in communities where rates of MRSA are high. These infections are most likely due to streptococci and coverage should focus on these bacteria. Concerns have been raised in the medical literature about empirical monotherapy with either trimethoprim-sulfamethoxazole or doxycycline in skin and soft-tissue infections. The anti-streptococcal activity of trimethoprimsulfamethoxazole and doxycycline has been described as "uncertain" [38].

Early data published at the time of FDA approval in 1973 indicated a very low MIC of $0.05 / 1 \mathrm{mcg} / \mathrm{ml}$ for the trimethoprim and sulfamethoxazole components, respectively [39]. Despite the impressive in vitro data, a randomized, double-blind study published in 1973 showed trimethoprim-sulfamethoxazole was inferior to penicillin $G$ in the treatment of group A streptococcal pharyngitis and tonsillitis [40]. A 1999 in vitro study by Kaplan of Streptococcus pyogenes isolates was discontinued early because of a high rate of resistance to trimethoprim-sulfamethoxazole [41]. A recent in vitro study evaluating trimethoprimsulfamethoxazole activity against Streptococcus pyogenes showed susceptibility was dependent on the media used for culture [42]. Contemporary prospective clinical studies of trimethoprimsulfamethoxazole in monomicrobial, streptococcal mediated skin and soft-tissue infections are nonexistent.

Current literature suggests monotherapy with beta lactams would be appropriate therapy for immunocompetent outpatients with non-suppurative forms of cellulitis. It may be reasonable to cover MRSA in patients with suppurative cellulitis if the prevalence is high in the community. However, should this recommendation apply to cases of suppurative cellulitis in patients with recent skin and softtissue infections caused by MSSA? Recent articles also suggest it may be reasonable to limit coverage for diabetics with diffuse, nonpurulent cellulitis not associated with an ulcer to monotherapy with beta lactams.

What about inpatients? The current IDSA recommendations only suggest "consider" 
MRSA coverage; they do not recommend it. Should you consider empirically covering for MRSA in inpatients with non-suppurative cellulitis? The microbiological literature does not indicate or even remotely suggest that most common community-acquired pathogens associated with inpatient cases are different from outpatient. Unfortunately, this question has also not been adequately addressed in terms of clinical data. The prospective Jeng trial evaluated inpatients and reported a high rate of success for beta lactams but had no comparator. Again, it may be reasonable to cover diffuse, nonpurulent cellulitis with beta lactams only. Could diabetics with non-suppurative infection of the lower extremities receive monotherapy with a beta lactam? It may be reasonable for those provided the skin is intact. Non-infected ulcers are unlikely to be associated with a surrounding cellulitis. The 2012 IDSA diabetic foot guidelines did not address this situation [38].

The current (2005) practice guidelines for management of SSTIs can be found at the IDSA website [43].

\section{ACKNOWLEDGMENTS}

No funding or sponsorship was received for this study or publication of this article. John Bowman is the guarantor for this article, and takes responsibility for the integrity of the work as a whole.

Conflict of interest. Michael Horseman and John Bowman have no conflicts of interest to disclose.

Compliance with ethics guidelines. This article does not contain any studies with human or animal subjects performed by any of the authors.
Open Access. This article is distributed under the terms of the Creative Commons Attribution Noncommercial License which permits any noncommercial use, distribution, and reproduction in any medium, provided the original author(s) and the source are credited.

\section{REFERENCES}

1. Gilbert DN. Sanford guide to antimicrobial therapy 2013. Sperryville, Va.: Antimicrobial therapy, 2013.

2. Johns Hopkins Antibiotics (ABX) Guide 2012. Bartlett J. http://www.hopkinsguides.com/hopkins/ ub/view/Johns_Hopkins_ABX_Guide/540106/all/ Cellulitis). Accessed May 22, 2013.

3. Stevens DL, Bisno AL, Chambers HF, et al. Practice guidelines for the diagnosis and management of skin and soft-tissue infections. Clin Infect Dis. 2005;41:1373-406.

4. Practice Guidelines for Skin and Soft Tissue Infections 2013. IDSA. http://www.idsociety.org/ Organ_System/). Accessed May 22, 2013.

5. Liu C, Bayer A, Cosgrove SE, et al. Clinical practice guidelines by the infectious diseases society of America for the treatment of methicillin-resistant Staphylococcus aureus infections in adults and children. Clin Infect Dis. 2011;52:e18-55.

6. Outpatient management of skin and soft tissue infections in the era of community-associated MRSA 2007. Centers for Disease Control. http:// www.cdc.gov/mrsa/pdf/Flowchart-k.pdf). Accessed Jun 3, 2013.

7. Moellering $\mathrm{M}$, Robert C Jr. The growing menace of community-acquired methicillin-resistant Staphylococcus aureus. Ann Intern Med. 2006;144: 368-70.

8. Pallin DJ, Binder WD, Allen MB, et al. Clinical trial: comparative effectiveness of cephalexin plus trimethoprim-sulfamethoxazole versus cephalexin alone for treatment of uncomplicated cellulitis: a randomized controlled trial. Clin Infect Dis. 2013;56:1754-62.

9. Chira S, Miller LG. Staphylococcus aureus is the most common identified cause of cellulitis: a systematic review. Epidemiol Infect. 2010;138:313-7.

10. Jeng A, Beheshti M, Li J, Nathan R. The role of betahemolytic streptococci in causing diffuse, 
nonculturable cellulitis: a prospective investigation. Medicine (Baltimore). 2010;89:217-26.

11. Daum RS. Clinical practice. Skin and soft-tissue infections caused by methicillin-resistant Staphylococcus aureus. N Engl J Med. 2007;357:380-90.

12. Chambers HF. Cellulitis, by any other name. Clin Infect Dis. 2013;56:1763-4.

13. Hirschmann JV, Raugi GJ. Lower limb cellulitis and its mimics: part I. Lower limb cellulitis. J Am Acad Dermatol. 2012;67:163. e1-12 (quiz 175-6).

14. Ki V, Rotstein C. Bacterial skin and soft tissue infections in adults: a review of their epidemiology, pathogenesis, diagnosis, treatment and site of care. Can J Infect Dis Med Microbiol. 2008;19:173-84.

15. Gunderson CG. Cellulitis: definition, etiology, and clinical features. Am J Med. 2011;124:1113-22.

16. Swartz MN. Cellulitis. N Engl J Med. 2004;350: 904-12.

17. Eells SJ, Chira S, David CG, Craft N, Miller LG. Nonsuppurative cellulitis: risk factors and its association with Staphylococcus aureus colonization in an area of endemic community-associated methicillin-resistant S. aureus infections. Epidemiol Infect. 2011;139: 606-12.

18. Rajan S. Skin and soft-tissue infections: classifying and treating a spectrum. Cleve Clin J Med. 2012;79:57-66.

19. Bailey E, Kroshinsky D. Cellulitis: diagnosis and management. Dermatol Ther. 2011;24:229-39.

20. Al-Niaimi F, Cox N. Cellulitis and lymphoedema: a vicious cycle. J Lymph. 2009;4:38-42.

21. Baddour LM. Cellulitis syndromes: an update. Int J Antimicrob Agents. 2000;14:113-6.

22. Bjornsdottir S, Gottfredsson M, Thorisdottir AS, et al. Risk factors for acute cellulitis of the lower limb: a prospective case-control study. Clin Infect Dis. 2005;41:1416-22.

23. Karppelin M, Siljander T, Vuopio-Varkila J, et al. Factors predisposing to acute and recurrent bacterial non-necrotizing cellulitis in hospitalized patients: a prospective case-control study. Clin Microbiol Infect. 2010;16:729-34.

24. Gabillot-Carre M, Roujeau JC. Acute bacterial skin infections and cellulitis. Curr Opin Infect Dis. 2007;20:118-23.

25. Phoenix G, Das S, Joshi M. Diagnosis and management of cellulitis. BMJ. 2012;345:e4955.
26. Duvanel T, Auckenthaler R, Rohner P, Harms M, Saurat JH. Quantitative cultures of biopsy specimens from cutaneous cellulitis. Arch Intern Med. 1989;149:293-6.

27. Baddour LM, Googe PB, Prince TL. Possible role of cellular immunity: a case of cellulitis. Clin Infect Dis. 2001;32:E17-21.

28. Perl B, Gottehrer NP, Raveh D, Schlesinger Y, Rudensky B, Yinnon AM. Cost-effectiveness of blood cultures for adult patients with cellulitis. Clin Infect Dis. 1999;29:1483-8.

29. Sadow KB, Chamberlain JM. Blood cultures in the evaluation of children with cellulitis. Pediatrics. 1998;101:E4.

30. Verma D, Chapnick E, Ghitan M, et al. The yield of blood cultures in community acquired cellulitis (CAC) (Poster: 546, Session: Dianostic Microbiology). In: Infectious Diseases Society of America 45th Annual Meeting October 4-7, 2007; San Diego, California; 2013. https://idsa.confex. com/idsa/2007/webprogram/Paper23663.htm). Accessed May 23, 2013.

31. Khawcharoenporn T, Tice A. Empiric outpatient therapy with trimethoprim-sulfamethoxazole, cephalexin, or clindamycin for cellulitis. Am J Med. 2010;123:942-50.

32. Gorwitz RJ. The role of ancillary antimicrobial therapy for treatment of uncomplicated skin infections in the era of community-associated methicillin-resistant Staphylococcus aureus. Clin Infect Dis. 2007;44:785-7.

33. Gunderson CG, Martinello RA. A systematic review of bacteremias in cellulitis and erysipelas. J Infect. 2012;64:148-55.

34. Madaras-Kelly KJ, Remington RE, Oliphant CM, Sloan KL, Bearden DT. Efficacy of oral betalactam versus non-beta-lactam treatment of uncomplicated cellulitis. Am J Med. 2008;121: 419-25.

35. Jenkins TC, Sabel AL, Sarcone EE, Price CS, Mehler PS, Burman WJ. Skin and soft-tissue infections requiring hospitalization at an academic medical center: opportunities for antimicrobial stewardship. Clin Infect Dis. 2010;51:895-903.

36. Jenkins TC, Knepper BC, Sabel AL, et al. Decreased antibiotic utilization after implementation of a guideline for inpatient cellulitis and cutaneous abscess. Arch Intern Med. 2011;171:1072-9.

37. Thomas KS, Crook AM, Nunn AJ, et al. Penicillin to prevent recurrent leg cellulitis. $\mathrm{N}$ Engl J Med. 2013;368:1695-703. 
38. Lipsky BA, Berendt AR, Cornia PB, et al. 2012 Infectious Diseases Society of America clinical practice guideline for the diagnosis and treatment of diabetic foot infections. Clin Infect Dis. 2012;54:e132-73.

39. Bushby SR. Trimethoprim-sulfamethoxazole: in vitro microbiological aspects. J Infect Dis. 1973;128 Suppl:442 (p 462).

40. Trickett PC, Dineen P, Mogabgab W. Clinical experience: respiratory tract. Trimethoprimsulfamethoxazole versus penicillin $G$ in the treatment of group A beta-hemolytic streptococcal pharyngitis and tonsillitis. J Infect Dis. 1973;128 Suppl:693 (p 695).
41. Kaplan EL, Johnson DR, Del Rosario MC, Horn DL. Susceptibility of group A beta-hemolytic streptococci to thirteen antibiotics: examination of 301 strains isolated in the United States between 1994 and 1997. Pediatr Infect Dis J. 1999;18: 1069-72.

42. Bowen AC, Lilliebridge RA, Tong SY, et al. Is Streptococcus pyogenes resistant or susceptible to trimethoprim-sulfamethoxazole? J Clin Microbiol. 2012;50:4067-72.

43. Current practice guidelines for management of SSTI's; 2005. http://cid.oxfordjournals.org/content/ 41/10/1373/F3.expansion.html. Accessed Oct 24, 2013. 